\title{
Research Paper: Association Between Physical Activity and Post menopausal Symptoms in Saudi Women: A Cross- sectional Study
}

\author{
Muneera Almurdi ${ }^{1}$ (D), Syamala Buragadda ${ }^{*}$ (D)
}

1. Department of Rehabilitation Health Sciences, College of Applied Medical Sciences, King Saud University, Riyadh, Saudi Arabia.

Cftation: Almurdi M, Buragadda B. Association Between Physical Activity and Post menopausal Symptoms in Saudi Women: A Cross-sectional Study. Iranian Rehabilitation Journal. 2021; 19(2):157-164. http://dx.doi.org/10.32598/irj.19.2.1237.1

dol'http://dx.doi.org/10.32598/irj.19.2.1237.1

(c) (i) (8)

Article info:

Received: 07 Oct 2020

Accepted: 20 Jun 2021

Available Online: 01 June 2021

Keywords:

Climacteric symptoms, Post menopause, Menopause,

Physical activity

\section{A BSTRACT}

Objectives: Menopause is the termination of a woman's menstrual cycle for a year. In this condition, women's health status declines due to hormonal changes and aging. Physical Activity (PA) is among the best available alternatives for managing menopause-related symptoms without any adverse effects. This study aimed to assess the relationship between PA and symptoms, such as fatigue, depression, and insomnia in Saudi menopausal women.

Methods: This cross-sectional study involved 60 menopausal women; they were divided into physically active and inactive groups. The group allocation was conducted based on their PA level determined by the International Physical Activity Questionnaire Short-Form (IPAQ-SF). Menopausal symptoms, such as fatigue, depression, and insomnia were assessed using the Fatigue Severity Scale (FSS), Beck Depression Inventory-II (BDI-II), and Insomnia Severity Index (ISI), respectively, in the study groups. The association between PA and fatigue, depression, and insomnia was assessed using the Pearson correlation coefficient. The significance level was set at $\mathrm{P} \leq 0.05$.

Results: Fatigue, depression, and insomnia were more prevalent in physically inactive post-menopause women than the active women. A significant difference was observed in the outcome variables between the study groups. Furthermore, a significant association was detected between PA and fatigue, depression, and insomnia in the study participants.

Discussion: Half of the explored menopausal women were physically active. Physically active menopausal women presented less fatigue, depression, and insomnia, compared to their inactive counterparts. This study suggested that PA positively impacted menopausal symptoms (fatigue, insomnia, \& depression). Besides, the collected results highlighted the importance of physical activity among menopausal women.

\footnotetext{
* Corresponding Author:

Syamala Buragadda, MSc.

Address: Department of Rehabilitation Health Sciences, College of Applied Medical Sciences, and King Saud University, Riyadh, Saudi Arabia.

Tel: +966 (53) 1262956

E-mail: syamala3110@yahoo.co.in
} 


\section{Highlights}

- Half of the studied menopausal women were physically active.

- Physically active menopausal women presented less fatigue, as well as low levels of depression and insomnia.

- Physical activity positively impacted menopausal symptoms (i.e., fatigue, insomnia, \& depression).

\section{Plain Language Summary}

Menopause is the end of a woman's menstrual cycle for one year. At this time, women's health declines due to hormonal changes and aging. Menopause usually occurs between 45 and 50 years of age and is considered a critical period in a women's life. The most common symptoms among menopausal women are vasomotor conditions, such as vaginal dryness, hot flashes, joints pain, insomnia, irritability, mood swings, and fatigue. Menopausal symptoms can be managed by hormone replacement therapy; however, exercise is among the best available alternative for managing menopause-related symptoms without adverse effects. Physical has many positive health benefits and is an effective way to prevent symptoms related to menopause. Evidence suggested beneficial physical activity effects; however, there is a lack of evidence on exercise and menopausal symptoms in Saudi women. This study aimed to examine the relationship between physical activity and menopause-related symptoms, such as fatigue, insomnia, and depression in Saudi women.

\section{Introduction}



enopause, an essential aspect of a women's life, is the termination of the menstrual cycle for a year and usually occurs between 45 and 50 years of age. It marks the end of the fertility period and women's health status declines due to hormonal changes and aging. It is usually associated with bodily changes, leading to biopsychological, hormonal, and emotional alternations [1]. The most frequent symptoms among $80 \%$ of menopausal women are vasomotor symptoms, such as night sweats, joints problems, and fatigue $[1,2]$. The age of natural menopause in Saudi women is 50 years. Besides, sociodemographic, reproductive, and lifestyle characteristics may affect menopausal symptoms [3]. Menopausal symptoms might be severe in women who do not exercise regularly [4].

Menopausal symptoms can be managed by hormone replacement therapy; however, exercise is among the best available alternatives for managing menopauserelated symptoms without adverse effects [5]. Physical Activity (PA) is any bodily movement produced by skeletal muscle contraction that requires energy expenditure. Furthermore, it provides numerous positive health benefits; it is also an effective approach to prevent symptoms related to menopause [6]. Depression, insomnia, and fatigue are some of the most prevalent menopauserelated symptoms. Numerous studies suggested that PA significantly reduces depression [7]; however, other studies found that exercise can only affect general conditions, such as biopsychosocial symptoms [7]. However, PA may not significantly influence sexual and vasomotor symptoms [8]. In the early years of menopause, women are highly vulnerable to depressive symptoms. Accordingly, clinicians should regularly consider screening women for any symptoms of depression or other disorders. Subsequently, immediate treatment should be initiated if necessary.

Another most troubling clinical manifestation among $50 \%-75 \%$ of menopausal women is sleep disturbance/ insomnia [9] that negatively impacts life quality [10]. There is a bidirectional relationship between insomnia and depression; insomnia can be a consequence of clinical depression or instigate mood swings [11]. In some women, decreased estrogen levels may generate hot flashes in the upper body; it may also result in fatigue that interferes with Daily Living Activities (DLAs). Women with signs of menstrual fatigue may experience irritability, as well as reduced attention and wakefulness. Furthermore, menopause-related fatigue may adversely affect mood, sleep, and Quality of Life (QoL) [12]. A retrospective study performed in China signified that depression, insomnia, and fatigue were prevalent among menopausal women [12].

Alquaiz et al. assessed the relationship between women's lifestyles and menopausal symptoms [3]. They concluded that obese and physically inactive females were more prone to experiencing severe menopausal symp- 
toms [3]. Evidence indicated the positive impact of PA on menopausal symptom severity; however, data concerning the relationship between physical activity and post-menopausal symptoms in Saudi women remain scarce. Thus, the present study aimed to examine the relationship between physical activity and menopauserelated symptoms, i.e., fatigue, depression, and insomnia in Saudi women.

\section{Methods}

This cross-sectional study was conducted from September 2019 to March 2020 in Riyadh City, Saudi Arabia. After obtaining ethical approval from the Institutional Review Board (IRB/E-2020), King Saud University, the study participants were virtually invited to participate in the study. The study inclusion criteria included menopausal women aged 46-65 years, who voluntarily agreed to participate in this research and could understand the questions to answer them. Women with a chronic illness who refrained from physical activity or currently under medication for any psychological illnesses were excluded from the study. All study participants were screened for the inclusion criteria. Moreover, the sociodemographic characteristics of the study participants were noted. Additionally, the study participants were requested to complete an online battery questionnaire, consisting of the following: the International Physical Activity Questionnaire- Short Form (IPAQ-SF), Fatigue Severity Scale (FSS), Beck Depression Inventory-II (BDI-II), and Insomnia Severity Index (ISI). All the respondents provided written informed consent forms and were requested to contact the researcher involved in the data collection if required.

The research participants were recruited via social media advertising, using platforms such as Snap Chat, Twitter, WhatsApp, and Facebook; however, the study sample was restricted to women aged 45-65 years. The survey included questions about women's age and history of the menstrual period (present, irregular, or absent). Then, each research participant received an online link that included 4 Questionnaires, as follows: The IPAQSF to assess physical activity level [Metabolic equivalents of Task (MET)], the FSS to measures the severity of fatigue, the BDI-II to assess depression, and the ISI to assess insomnia. The study sample was divided into physically active ( $>600$ MET-minutes/week) and inactive groups ( $<600$ MET-minutes/week) based on the physical activity level. There was no difference in age, area of residence, and educational level between the research groups.
The IPAQ-SF (Arabic version) assessed leisure-time PA, including DLAs and sitting time. It assesses active individuals in the past 7 days and found it a helpful tool [13]. Walking, moderate-intensity activities and vigorous-intensity activities are specific types of assessed activities. The frequency was measured in days per week, and duration was examined as time spent per day. They are separately measured per domain. PA was divided into categorical scores in this study; physically-inactive and physically-active groups [14].

The FSS is a self-report questionnaire to evaluate the severity of fatigue in various disorders. We used FSSAr (Arabic version) to explore fatigue, i.e., considered a valid and reliable tool for fatigue assessment in the Arabic population [15]. It includes 9 items, scored on a Likert-type scale, ranging from 1 (strongly disagree) to 7 (strongly agree). The average score of all items ranges between 1 (minimum) and 7 (maximum). The cut-off Mean \pm SD values for healthy individuals equals $2.3 \pm 0.7$, and a score of $\geq 4$ is indicative of fatigue [16].

We used the BDI-II questionnaire (Arabic version) to assess the symptoms of depression. Moreover, it consists of 21 items, i.e., scored from 0 to 3 . A higher score indicates severe depression [17]. It is a valid measure to assess the depression severity among the Arabic population [18].

The ISI (Arabic version) investigated any sleep disturbance symptoms with 7 domains, scored from 0 to 4 , with scores ranging from 0 to 28; the higher the scores, the greater the sleep disturbance. The modified ISI Arabic version is a valid and reliable measure of insomnia and is widely used in the Arabic population [19].

The obtained data were analyzed using SPSS. The between-group differences were analyzed using an Independent Samples t-test. The association between physical activity and menopausal symptoms (insomnia, depression, fatigue) was analyzed using Pearson's correlation coefficient.

\section{Results}

After excluding the incomplete data from a sample of 104, 60 subjects were included for the final data analysis. Based on the study participant's level of physical activity, they were grouped as physically active and physically inactive. Each research group consisted of 30 participants. Furthermore, the Mean \pm SD age of the active and inactive groups was $52.8 \pm 4.0$ and $53.3 \pm 4.39$ years, respectively. No significant difference was observed 
Table 1. Clinical characteristics of the study participants $(\mathrm{N}=60)$

\begin{tabular}{|c|c|c|c|c|}
\hline \multicolumn{2}{|r|}{ Variables } & $\begin{array}{l}\text { Physically-Active Group } \\
\qquad(\mathrm{n}=\mathbf{3 0})\end{array}$ & $\begin{array}{l}\text { Physically-Inactive } \\
\text { Group }(n=30)\end{array}$ & $\mathbf{P}$ \\
\hline \multicolumn{2}{|r|}{ Age (y), Mean $\pm S D$} & $52.8 \pm 4.0$ & $53.3 \pm 4.39$ & 0.60 \\
\hline \multirow{3}{*}{ Area of residence } & Central region & 21 & 22 & \multirow{3}{*}{0.52} \\
\hline & & & & \\
\hline & Other parts of Riyadh & 09 & 08 & \\
\hline \multirow{3}{*}{ Educational level } & Below high school & 16 & 18 & \multirow{3}{*}{0.32} \\
\hline & & & & \\
\hline & High school and above & 14 & 12 & \\
\hline \multirow{2}{*}{$\begin{array}{c}\text { Level of PA (MET-min/ } \\
\text { week) }\end{array}$} & Min-Max & $700-1490$ & $340-590$ & \multirow{2}{*}{$0.0001^{*}$} \\
\hline & Mean $\pm S D$ & $1228.1 \pm 34.7$ & $503.4 \pm 13.1$ & \\
\hline \multirow{5}{*}{ Fatigue } & No $(n=22)$ & $16(72.7)$ & $6(27.2)$ & \multirow{5}{*}{$0.0001^{*}$} \\
\hline & Yes $(n=38)$ & $14(36.8)$ & $24(63.1)$ & \\
\hline & & & & \\
\hline & Min-Max & $7-54$ & $18-63$ & \\
\hline & Mean $\pm S D$ & $34.4 \pm 3.2$ & $48.9 \pm 2.3$ & \\
\hline \multirow{7}{*}{ Depression } & $0-10$ (no depression) $(n=21)$ & $16(76.1)$ & $5(23.9)$ & \multirow{7}{*}{$0.0001^{*}$} \\
\hline & $11-16$ (mild depression) $(n=8)$ & $6(75)$ & $2(25)$ & \\
\hline & $17-20$ (borderline depression) $(n=7)$ & $3(42.8)$ & $4(57.1)$ & \\
\hline & 21-30 (moderate depression) $(n=16)$ & $4(25)$ & $12(75)$ & \\
\hline & $31-40$ (severe depression) $(n=8)$ & $1(12.5)$ & $7(87.5)$ & \\
\hline & Min-Max & $2-16$ & $17-39$ & \\
\hline & Mean+SD & $8.7+0.7$ & $278+12$ & \\
\hline \multirow{8}{*}{ Insomnia } & 0-7 (no insomnia) $(n=16)$ & $10(62.5)$ & $6(37.5)$ & \multirow{8}{*}{$0.0001^{*}$} \\
\hline & 8-14 (sub threshold insomnia) $(n=26)$ & $15(57.6)$ & $11(42.3)$ & \\
\hline & $15-21$ (moderate insomnia) $(n=12)$ & $4(33.3)$ & $8(66.6)$ & \\
\hline & & & & \\
\hline & $22-28$ (severe insomnia) $(n=6)$ & $1(16.6)$ & $5(83.3)$ & \\
\hline & Min-Max & $4-27$ & $5-28$ & \\
\hline & & & & \\
\hline & Mean $\pm S D$ & $11.0 \pm 0.98$ & $14.9 \pm 1.2$ & \\
\hline
\end{tabular}

${ }^{*} \mathrm{P} \leq 0.05$.

Iranian Rehabilitation Journal

Pearson correlation coefficient data revealed that PA was negatively associated with depression $(\mathrm{r}=-0.9 ; \mathrm{P} \leq 0.05)($ Figure 1$)$, insomnia ( $\mathrm{r}=-0.6 ; \mathrm{P} \leq 0.05)$ (Figure 2), and fatigue $(\mathrm{r}=-0.65 ; \mathrm{P} \leq 0.05)$ (Figure 3 ).

in the age range between the study groups. More than $60 \%$ of the inactive group participants presented fatigue, while $72.7 \%$ of the active participants reported no fatigue. In total, 8 participants reported severe depression on the BDI-II, out of which, 7(87.5\%) subjects belonged to the inactive group.
Similarly, 6 of the explored menopausal women reported severe insomnia on the ISI; of whom, $5(83.3 \%)$ were in the physically inactive group. A significant difference was observed in fatigue, depression, and insomnia between the study groups $(\mathrm{P} \leq 0.05)$ (Table 1$)$. 


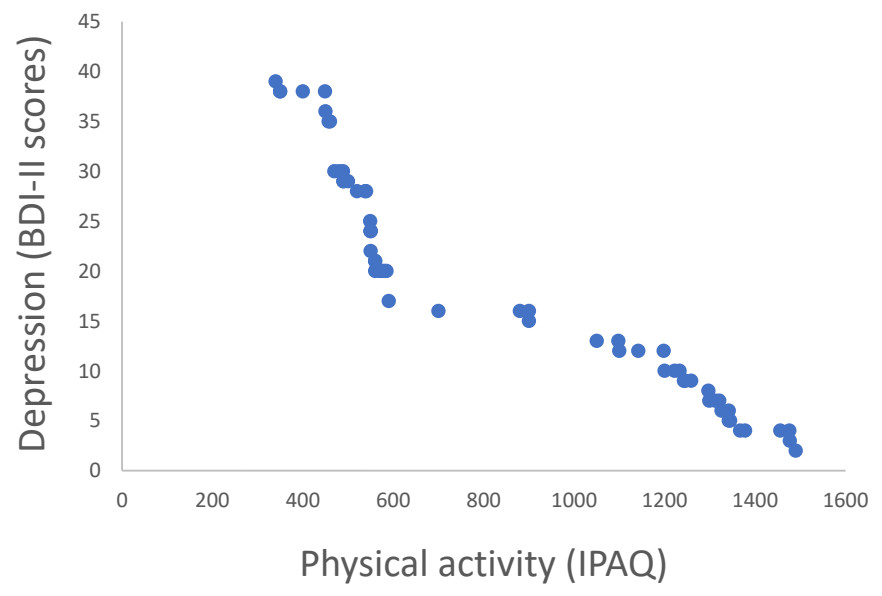

Figure 1. Association between physical activity and depression in the study participants

Iranian Rehabilitation Journal

\section{Discussion}

The current study investigated the relationship between PA and menopausal symptoms, including fatigue, depression, and insomnia in women living in Riyadh. Half of the explored menopausal women were physically active. Besides, there was a significant relationship in the study variables between the study groups. This crosssectional study signified that PA positively impacted menopausal symptoms (fatigue, insomnia, depression). Moreover, and the collected results highlighted the importance of PA among menopausal women.

The study participants who obtained a score $>4$ on the fatigue scale were considered as experiencing fatigue. We found that fatigue was higher among physically inactive women. This finding was consistent with another study (2017), which examined the prevalence of menopausal symptoms, its effect on the QoL, and perception on obtaining treatment in a multiracial community of postmenopausal women in Klang Valley, Malaysia. The relevant data obtained by face-to-face interviews suggested that fatigue $(59.3 \%)$ was a commonly reported symptom in menopausal women; $73.3 \%$ of the examined women reported joint and muscular discomfort [20]. We also found a significant relationship between PA and such menopausal symptoms as, depression and insomnia. This result was in line with those of a similar study that included 370 Brazilian menopause women aged 40-65 years. The study indicated that menopausal symptoms were less observed in middle-aged women who exercised. There was a significant difference in menopausal symptoms between the groups with different physical activity levels [21]. Another cross-sectional study also demonstrated similar physical activity and anxiety results in menopausal women assessed using the Goldenberg's questionnaire and the IPAQ. The authors argued that physical activity positively influenced physical health, social functioning, anxiety, and depression in menopausal women [22].

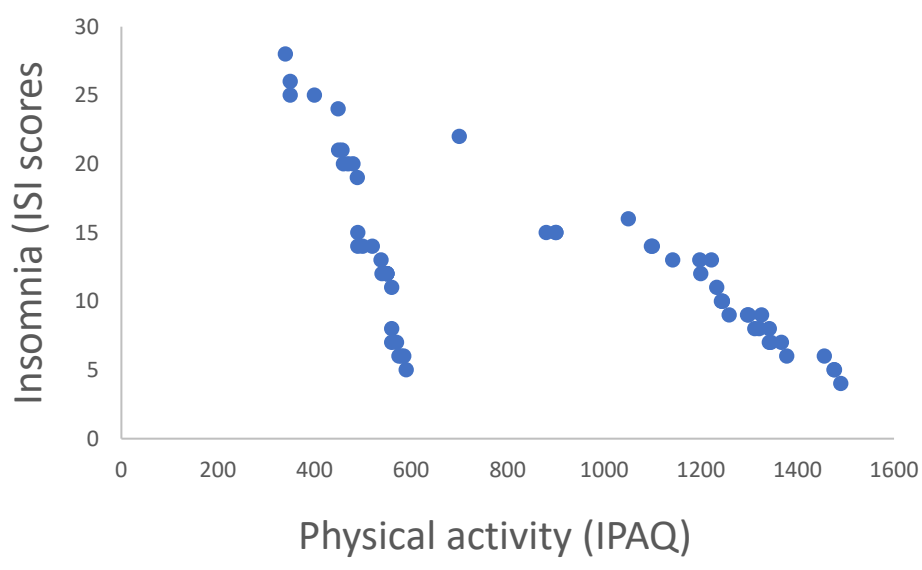

Figure 2. Association between physical activity and insomnia in the study subjects 


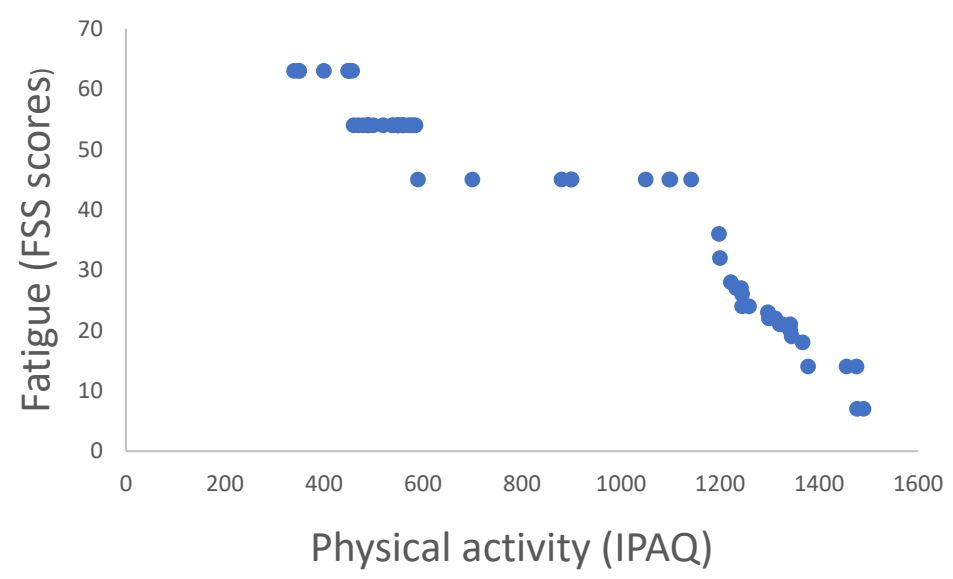

Figure 3. Association between physical activity and fatigue in the study sample

A woman in the menopausal period is at high risk of developing a specific medical condition, as an associated complication, including cardiovascular disease, osteoporosis, urinary incontinence, weight gain, and sexual dysfunction due to vaginal dryness [7]. Previous research reported that the habitual practice of at least moderateintensity PA for 60 minutes per day seems to beneficially affect menopausal symptoms and QOL, especially on psychosocial domains. The effects of habitual PA at the psychological level seem to be at least partially correlated with decreased menopausal symptoms and weight loss [23]. A randomized controlled trial on 164 women reported that walking and yoga enhance mood and positively affect the menopause-related QoL [24]. Another interventional study involving 248 post-menopausal sedentary women documented that aerobic exercises improved insomnia and depression in them; however, there was no significant improvement in sleep quality with exercise training [25].

Similar to our study findings, a cross-sectional cohort study among Taiwanese menopause women suggested the positive impact of PA on menopausal symptoms [21]. The literature on PA in menopausal women in Riyadh is scarce. Thus, our study may add value to the existing data. There were a few limitations worth mentioning in this study. The cross-sectional nature was the study's main limitation, as the cause and effect relationship between PA and menopausal symptoms, such as fatigue, depression, and insomnia could not be established. Second, our study included a small number of menopausal women living in Riyadh. This was because of two reasons; most Saudi women were unwilling to answer all the questions related to menopause due to social stigma, and lockdown due to COVID-19 refrained the authors from further continuing the study.
Furthermore, we conducted online sample recruitment and limited it to Riyadh; we also used self-report questionnaires which may have generated recall biases. Future studies with a large sample size involving menopausal women from different regions of the Saudi Kingdom and objective PA assessments to establish a strong relationship between PA and menopausal symptoms are highly recommended. This may help to develop interventions to effectively treat menopausal symptoms.

\section{Conclusion}

Physically active Saudi women presented less depression, insomnia, and fatigue, compared to their inactive counterparts. Healthcare professionals may help women reduce their menopausal symptoms by encouraging them to be physically active.

\section{Ethical Considerations}

\section{Compliance with ethical guidelines}

This study was approved by the Ethics Committee of the Institutional Review Board (IRB/E-2020), King Saud University.

Funding

This research did not receive any grant from funding agencies in the public, commercial, or non-profit sectors.

\section{Authors' contributions}

Conceptualization, Methodology, and Investigation: Muneera Almurdi, Syamala Buragadda; Writing - review \& editing: Muneera Almurdi, Syamala Buragadda; 
Resources: Muneera Almurdi, Syamala Buragadda; Supervision: Muneera Almurdi, Syamala Buragadda.

\section{Conflict of interest}

The authors have no conflict of interest to declare.

\section{Acknowledgments}

The authors extend their appreciation to the Deanship of Scientific Research, College of Applied Medical Sciences Research Centre at King Saud University, Riyadh. We are indebted to our students Ms. Norah alhogail, Ms. Rawan Zahrani, Ms. Yasmeen Almutlaq, Ms. Hadeel alshammari for their valuable contribution in data collection and support in successful completion of the study.

\section{References}

[1] AlDughaither A, AlMutairy H, AlAteeq M. Menopausal symptoms and quality of life among Saudi women visiting primary care clinics in Riyadh, Saudi Arabia. International Journal of Women's Health. 2015; 7:645-53. [DOI:10.2147/ IJWH.S84709] [PMID] [PMCID]

[2] Ogwumike OO, Kaka B, Adegbemigun O, Abiona T. Healthrelated and sociodemographic correlates of physical activity level amongst urban menopausal women in Nigeria. Maturitas. 2012; 73(4):349-53. [DOI:10.1016/j.maturitas.2012.09.010] [PMID]

[3] Alquaiz JM, Siddiqui AR, Tayel SA, Habib FA. Determinants of severity of menopausal symptoms among Saudi women in Riyadh city. Climacteric. 2014; 17(1):71-8. [DOI:10.3109/13697 137.2013.815161] [PMID]

[4] Kim MJ, Cho J, Ahn Y, Yim G, Park HY. Association between physical activity and menopausal symptoms in perimenopausal women. BMC Women's Health. 2014; 14:122. [DOI:10.1186/1472-6874-14-122] [PMID] [PMCID]

[5] Daley A, MacArthur C, McManus R, Stokes-Lampard H, Wilson S, Roalfe A, et al. Factors associated with the use of complementary medicine and non-pharmacological interventions in symptomatic menopausal women. Climacteric. 2006; 9(5):336-46. [DOI:10.1080/13697130600864074] [PMID]

[6] Villaverde-Gutiérrez C, Araujo E, Cruz F, Roa JM, Barbosa W, Ruíz-Villaverde G. Quality of life of rural menopausal women in response to a customized exercise programme. Journal of Advanced Nursing. 2006; 54(1):11-9. [DOI:10.1111/ j.1365-2648.2006.03784.x] [PMID]

[7] Bromberger JT, Epperson CN. Depression during and after the perimenopause: Impact of hormones, genetics, and environmental determinants of disease. Obstetrics and Gynecology Clinics of North America. 2018; 45(4):663-78. [DOI:10.1016/j.ogc.2018.07.007] [PMID] [PMCID]

[8] Haimov-Kochman R, Constantini N, Brzezinski A, HochnerCelnikier D. Regular exercise is the most significant lifestyle parameter associated with the severity of climacteric symptoms: a cross sectional study. European Journal of Obstetrics \& Gynecology and Reproductive Biology. 2013; 170(1):229-34 [DOI:10.1016/j.ejogrb.2013.06.018] [PMID]

[9] Dobkin RD, Menza M, Bienfait KL, Allen LA, Marin H, Gara MA. Ramelteon for the treatment of insomnia in menopausal women. Menopause International. 2009; 15(1):13-8. [DOI:10.1258/mi.2009.009002] [PMID] [PMCID]

[10] Bolge SC, Balkrishnan R, Kannan H, Seal B, Drake CL. Burden associated with chronic sleep maintenance insomnia characterized by nighttime awakenings among women with menopausal symptoms. Menopause. 2010; 17(1):80-6 [DOI:10.1097/gme.0b013e3181b4c286] [PMID]

[11] Brown JP, Gallicchio L, Flaws JA, Tracy JK. Relations among menopausal symptoms, sleep disturbance and depressive symptoms in midlife. Maturitas. 2009; 62(2):184-9. [DOI:10.1016/j.maturitas.2008.11.019] [PMID] [PMCID]

[12] Ruan X, Cui Y, Du J, Jin F, Mueck AO. Prevalence of climacteric symptoms comparing perimenopausal and postmenopausal Chinese women. Journal of Psychosomatic Obstetrics and Gynecology. 2017; 38(3):161-9. [DOI:10.1080/0167 482X.2016.1244181] [PMID]

[13] Garashi NH, Al Kandari JR, Ainsworth BE, Barac-Nieto M. Weekly physical activity from IPAQ (Arabic) recalls and from IDEEA activity meters. Health. 2020; 12(6):598-611. [DOI:10.4236/health.2020.126045]

[14] Committee IR. Guidelines for data processing and analysis of the International Physical Activity Questionnaire (IPAQ)short and long forms[Internet]. 2005 [Updated 2005 November] http:// www ipaq ki se/scoring pdf. 2005

[15] Al-Sobayel HI, Al-Hugail HA, AlSaif RM, Albawardi NM Alnahdi AH, Daif AM, et al. Validation of an Arabic version of fatigue severity scale. Saudi Medical Journal. 2016; 37(1):73-8. [DOI:10.15537/smj.2016.1.13055] [PMID] [PMCID]

[16] Dittner AJ, Wessely SC, Brown RG. The assessment of fatigue: A practical guide for clinicians and researchers. Journal of Psychosomatic Research. 2004; 56(2):157-70. [DOI:10.1016/ S0022-3999(03)00371-4]

[17] Al-Eisa E, Buragadda S, Melam GR. Association between physical activity and psychological status among Saudi female students. BMC Psychiatry. 2014; 14:238. [DOI:10.1186/ s12888-014-0238-3] [PMID] [PMCID]

[18] Alansari BM. Internal consistency of an Arabic adaptation of the Beck Depression Inventory-II with college students in eighteen Arab countries. Social Behavior and Personality. 2006; 34(4):425-30. [DOI:10.2224/sbp.2006.34.4.425]

[19] Suleiman KH, Yates BC. Translating the insomnia severity index into Arabic. Journal of Nursing Scholarship. 2011; 43(1):49-53. [DOI:10.1111/j.1547-5069.2010.01374.x] [PMID]

[20] Abdullah B, Moize B, Ismail BA, Zamri M, Mohd Nasir NF. Prevalence of menopausal symptoms, its effect to quality of life among Malaysian women and their treatment seeking behaviour. The Medical Journal of Malaysia. 2017; 72(2):94-9. [PMID]

[21] Canário AC, Cabral PU, Spyrides MH, Giraldo PC, Eleutério J Jr, Gonçalves AK. The impact of physical activity on menopausal symptoms in middle-aged women. International Journal of Gynecology and Obstetrics. 2012; 118(1):34-6. [DOI:10.1016/j.ijgo.2012.02.016] [PMID] 
[22] Sharifi N, Jalili L, Khazaeian S, Nia AN. The Relationship between Physical Activity and General Health among Menopausal Women in Ahvaz, Iran. Electron Physician. 2017; 9(1):3639-45. [DOI:10.19082/3639] [PMID] [PMCID]

[23] de Azevedo Guimarães AC, Baptista F. Influence of habitual physical activity on the symptoms of climacterium/ menopause and the quality of life of middle-aged women. International Journal of Women's Health. 2011; 3:319-28. [DOI:10.2147/IJWH.S24822] [PMID] [PMCID]

[24] Elavsky S, McAuley E. Physical activity and mental health outcomes during menopause: A randomized controlled trial. Annals of Behavioral Medicine. 2007; 33:132-42. [DOI:10.1007/BF02879894] [PMID]

[25] Sternfeld B, Guthrie KA, Ensrud KE, LaCroix AZ, Larson JC, Dunn AL, et al. Efficacy of exercise for menopausal symptoms: A randomized controlled trial. Menopause. 2014; 21(4):330-8. [DOI:10.1097/GME.0b013e31829e4089] [PMID] [PMCID] 